ИЗВЕСТИЯ АКАДЕМИИ НАУК ЭСТОНСКОН ССР. ТОМ ХV СЕРИЯ ФИЗИКО-МАТЕМАТИЧЕСКИХ И ТЕХНИЧЕСКИХ НАУК. 1966, № II

\title{
ЭНЕРГЕТИЧЕСКИЙ ЭКВИВАЛЕНТ СОБСТВЕННЫХ ШУМОВ НЕКОТОРЫХ ФОТОЭЛЕКТРОННЫХ УМНОЖИТЕЛЕЙ
}

При регистрации малых $\beta$-активностей необходимо достичь максимально низкого аппаратурного фона (порядка $10 \mathrm{umn/мuн)} \mathrm{самой} \mathrm{счетной} \mathrm{установки.} \mathrm{Главным} \mathrm{источ-}$ ником фона сцинтилляционных счетчиков, предназначенных для измерения низкоэнергетического излучения, являются собственные шумы фотоэлектронных умножителей (ФЭУ). Среди основных причин, вызывающих появление собственных шумов ФЭУ, можно отметить следующие [1]:

1) термоэлектронная эмиссия фотокатода и первых динодов;

2) ток утечки;

3) ионная и оптическая обратная связь;

4) холодная (автоэлектронная) эмиссия.

Основная часть фона фотоумножителей возникает под действием термоэлектроноз: с фотокатода и первых динодов. Принято считать, что у сурьмяно-цезиевых фотокатсдов величина термоэлектронной эмиссии составляет порядка $10^{4}-10^{5}$ электронов в секунду с квадратного сантиметра поверхности при комнатной температуре:

С понижением температуры фотокатода уменьшается термоэлектронная эмиссия фотокатода и число фоновых импульсов. Поэтому некоторые авторы [2,3] при регистрации низких $\beta$-активностей (естественные $\mathrm{C}^{14}, \mathrm{H}^{3}$ ) сцинтилляционным методом применяли: охлаждение фотоумножителей.

Другой причиной возникновения шумов является ток утечки через изоляцию между анодом и другими электродами. Это чаще всего обусловлено оседанием тонкой пленкіт цезия на внутренней поверхности изоляторов ФЭУ, а также загрязнением наружной поверхности колбы и цоколя фотоумножителей. Обычно фоновые импульсы, связанные с наличием тока утечки, имеют малую амплитуду и ими можно пренебречь.

При повышенных напряжениях ФЭУ часто наблюдается резкое возрастание фона, обусловленное появлением ионной и оптической обратной связг между псследними динодами умножителя и катодом, а также возникновеннем автоэлектронной эмиссии с острых краев электродов.

Ионная обратная связь обусловлена положительными понами, возникающими в: анодной части умножителя при столкновении электронов с атомами остаточного газа и парами цезия. Ионы, ускоренные электрическим полем, бомбардируют катод и первые диноды, что вызывает возникновение вторичной фоновой электронной эмиссии.

Оптическая обратная связь, или так наз. световой темновой ток, вөзникает, когда электроны в анодной части фотоумножителя вызывают люминесценцию последних динодов и стекла колбы ФЭУ. При этом часть света люминесценции, достигая фотокатода, вызывает эмиссию фотоэлектронов, которые создают шумовые импульсы. С увеличением напряжения увеличивается энергия ионов и величина люминесцентного свечения тем самым увеличивается и значение фона. Поэтому при счете $\mathrm{C}^{14}$ для питания фота- 
умножителей обычно используют пониженное напряжение, что позволяет получить лучшее отношение сигнала к фону, увеличить стабильность и надежность рабюты ФЭУ [4].

Кроме вышеуказанных источников, причиной фоновых импульсов могут быть черенковское свечение стекла ФЭУ, вызванное космическим излучением, и люминесценция стекла колбы фотоумножителя, возникающая под действием радиоактивного распада калия $\left(\mathrm{K}^{40}\right)$, содержащегося часто в стекле ФЭУ.

Уменьшение аппаратурного фона, вызванное собственными шумами фотоумножителей, обычно достигается следующими методами:

1) нспользованием схемы совпадения;

2) охлаждением фотоумножителей;

3) тщательным отбором ФЭУ.

В данной работе применялся именно метод отбора ФЭУ, позволяющий из определенного количества ФЭУ относительно быстро отобрать экземпляры с наименьшими собственными шумами. Для этого измерялись следующие свойства различных типов ФӘУ:

а) энергетический эквивалент собственных шумов при скорости счета 10 имп/лин, который выражался в кило-электрон-вольтах по шкале: $\mathrm{NaJ}(\mathrm{Tl})$;

б) зависимость собственных шумов от напряжения;

в) амплитудное распределение шумовых импульсов.

\section{Аппаратура}

Все измерения проводились при помощи одноканальной сцинтилляционно̆ установки, блок-схема которой изображена на рис. 1.

Рис. 1. Блок-схема одноканальноӥ сцинтилляционной установки: 1 - стабилизированный выпрямитель высокого напряжения для питания ФЭУ; 2 - фотоэлектронный умножитель; 3 - предусилитель; 4 - главный усилитель; 5 - одноканальный амплитудный анализа. тор; 6 - пересчетный прибор; 7 - механический счетчик; 8 ртутный экран для защиты от космического излучения.

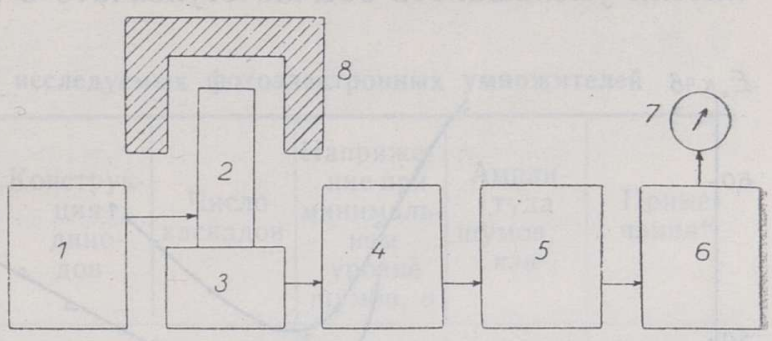

Установка собрана в основном из стандартных блоков шотландской фирмы «Nuclear Enterprises», краткая характеристика которых следуюцая:

1. Стабилизированный выпрямитель высокого напряжения (тип NE 5302), предназначенный для питания ФЭУ. При 24-часовом периоде работы общая нестабильность выходного напряжения не более $0,01 \%$. Возможно ступенчатое и плавное регулирование напряжения в пределах от 500 до 1500 в.

2. Неперегружаемый линейный импульсный усилитель состоит из предусилителя (тип NE 5202 А) и главного усилителя (тип NE 5202). Максимальное усиление его 50000 раз. Коэффициент нелинейных искажений и нестабильности меньше $0,25 \%$. Возможно ступенчатое и плавное регулирование усиления.

3. Одноканальный амплитудный анализатор (тип NE 5102), применяемый как в дифференциальном, так и в интегральном режиме. Oй 
имеет порог дискриминации от 3 до 100 в, регулируемый с точностью до 0,1 в; ширина его канала, регулируемая с точностью в 0,01 в, составляет от 0 до 10 в. Нестабильность амплитуды и ширины окна в течение суток равны соответственно 0,05 и 0,01 в.

Для счета импульсов использовался пересчетный-прибор ПС-64.

Стабилизация сетевого напряжения осуществлялась при помощи электронного стабилизатора (тип ST 2000) чехословацкого производства.

Делители напряжения для каждого типа ФЭУ смонтированы таким образом, чтобы обеспечить паспортный спектрометрический режим.

Для защиты от.космического излучения при измерении шумовых импульсов использовался ртутный экран (толщина слоя ртути $2,5 \mathrm{~cm}$ ).

\section{Экспериментальная часть}

Уровень собственных шумов каждого экземпляра ФЭУ устанавливался интегральным дискриминатором при пороге дискриминации $10 \mathrm{~b}$. изменяя коэффициент усиления главного усилителя. Таким образом, на выходе дискриминатора была достигнута скорость счета шумовых импульсов 10 имn/мuн. Энергетический эквивалент уровня собственных шумов выражен в шкале $\mathrm{NaJ}(\mathrm{Tl})$ (использовался стандартный спектрометрический кристалл $30 \times 10$ мм). В качестве опорной энергетической точки был выбран максимум фотолинии $\mathrm{Eu}^{155}$ с энергией 87 кэв. Максимум устанавливался путем снятия $\boldsymbol{\gamma}$-спектра указанного препарата дифференциальным дискриминатором при ширине канала в 1 в. Чтобы максимум попал в область дискриминатора $40-80$ в, усиление главного усилителя уменьшалось обычно ступенчато в $2-5$ раз.

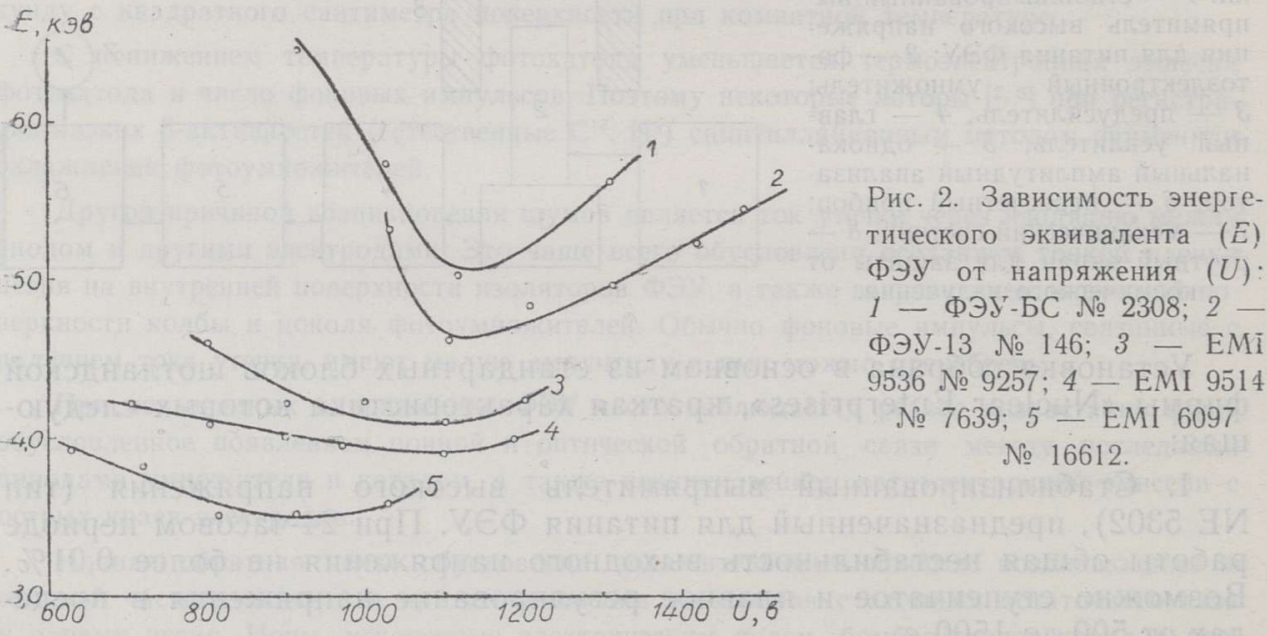

На рис. 2 изображена зависимость уровня собственных шумов некоторых типов ФӘУ от приложенного напряжения. Из хода кривых следует, что при увеличении напряжения уровень собственных шумов несколько падает и, пройдя минимум, снова возрастает. В нисходящей части кривых разность потенциалов между фотокатодом и первыми динодами, по-видимому, недостаточна, чтобы каждый фотоэлектрон вызвал появление лавины электронов. 
Д. Флейшман [5], исходя из статистических соображений, объясняет это явление возрастанием относительной флуктуации амплитудного распределения шумовых импульсов из-за уменьшения коэффициента вторичной эмиссии динодов при низких напряжениях на ФЭУ. Резкое возрастание шумов при увеличении напряжения $\mathrm{v}$ некоторых экземпляров ФӘУ் объясняется возникновением ионной и оптической обратной связи, а также автоэлектронной эмиссии. Следует отметить, что в пределах исследуемых напряжений уровень собственных шумов ФЭУ фирмы «ЕМІ» относительно мало зависит от приложенного напряжения.

Рис. 3. Интегральные спектры амплитуды собственных шумов некоторых ФЭУ: 1 ФЭУ-1С № 5901; 2 - ФЭУ-11 № 8301; 3 ФЭУ-1C № 5798; 4 - EMI 6097 № 16612 .

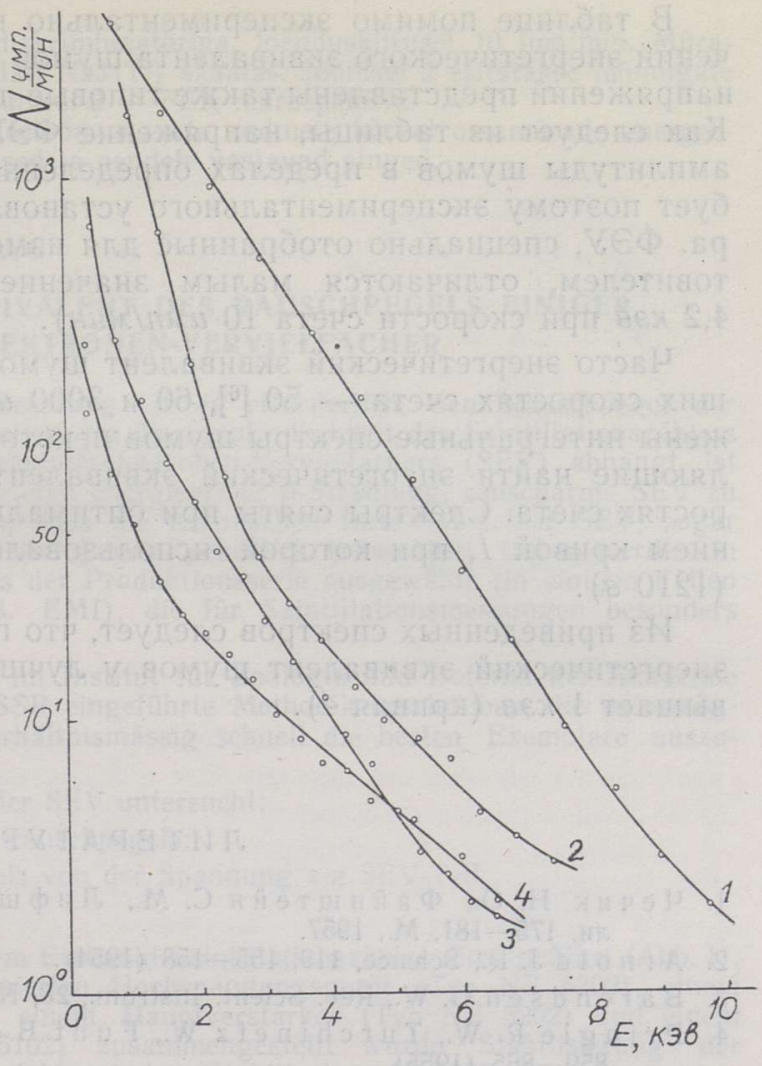

Характеристики и параметры исследуемых фотоэлектронных умножителей

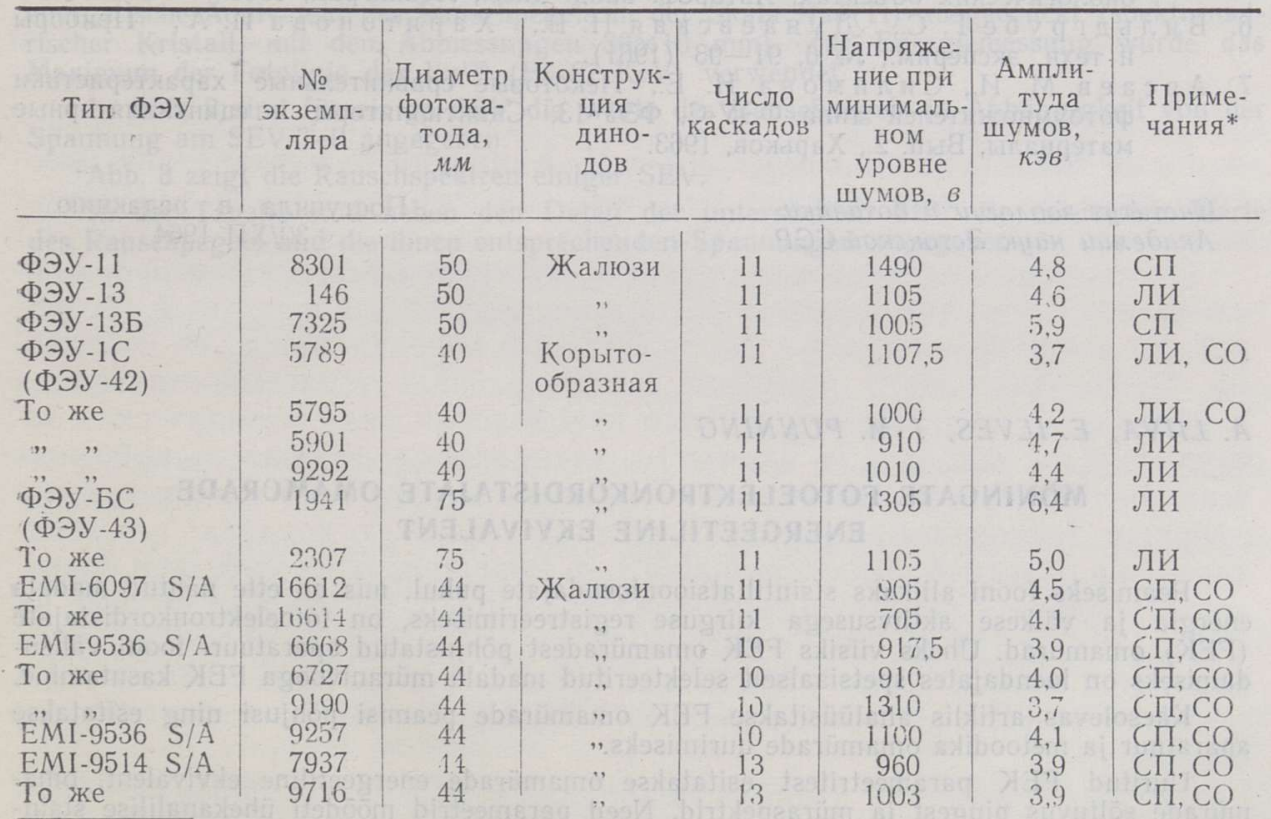

* СП - ФЭУ сернйного производства; ЛИ - ФЭУ лабораторного изготовления; СО - ФЭУ, специально отобранный заводом-изготовителем. 
В таблице помимо экспериментально найденных минимальных значений энергетического эквивалента шумов (в кәв) и соответствующих им напряжений представлены также типовые данные всех исследуемых ФЭУ. Как следует из таблицы, напряжение ФЭУ при минимальном значении амплитуды шумов в пределах определенного типа не постоянно и требует поэтому экспериментального установления для каждого экземпляра. ФЭУ, специально отобранные для измерения $\mathrm{C}^{14}$ и $\mathrm{H}^{3}$ заводом-изготовителем, отличаются малым значением амплитуды шумов $(3,5-$ 4,2 кәв при скорости счета 10 имл/мuн).

Часто энергетический эквивалент шумов ФЭУ выражается при больших скоростях счета $-50\left[{ }^{6}\right], 60$ и 3000 имn/мин [7]. На рис. 3 изображены интегральные спектры шумов некоторых экземпляров ФЭУ, позволяющие найти энергетический эквивалент шумов при различных скоростях счета. Спектры сняты при оптимальном напряжении за исключением кривой 1 , при которой использовалось повышенное напряжение (1210 в).

Из приведенных спектров следует, что при скорости счета 50 uмn/мuн энергетический эквивалент шумов у лучших экземпляров ФЭУ не превышает 1 кэв (кривая 4).

\section{Л И Т Е Р А Т У Р А}

1. Ч ечик Н. О., Файнштейн С. М., Лифшиц Т. М., Электронные умножители, 175-181, М., 1957.

2. A r nold J. R., Science, 119, 155-158 (1954).

3. B a r e nd s e n G. W., Rev. Scient. Instrum., 28, No. 6, 430-433 (1957).

4. Pringle R. W., Turchinetz W., Funt B. L., Rev. Scient. Instrum., 26, No. 9, $859-865$ (1955).

5. Ф ле й м а н Д. Г., Некоторые вопросы сцинтилляционного метода радиометрин и его применение для анализа малых количеств радиоактивных изотопов в биологических объектах, Автореф. канд. дисс., Ленинград, 1963.

6. Вильдгрубе Г. С., Дунаевская Н. В., Х аритонова И. А, Приборы и техн. эксперим., № 6, 91-93 (1961).

7. А рса ев М. И., Сил и мов а Н. Е., Некоторые сравнительные характеристики фотоумножителей типа ФЭУ-С, ФЭУ-13, Сцинтилляторы и сцинтилляторные материалы, Вып. 2., Харьков, 1963.

Ннститут зоологии и ботаники

Академии наук Эстонской ССР

Поступила в редакцию 30/XII 1964

A. LIIVA, E. ILVES, J.-M. PUNNING

\section{MÕNINGATE FOTOELEKTRONKORDISTAJATE OMAMURADE ENERGEETILINE EKVIVALENT}

Peamiseks fooni allikaks stsintillatsioonloendajate puhul, mis on ette nähtud madala energia ja väikese aktiivsusega kiirguse registreerimiseks, on fotoelektronkordistajate (FEK) onlamürad. Uheks viisiks FEK omamüradest põhjustatud aparatuuri fooni vähendamiseks on loendajates spetsiaalselt selekteeritud madala müranivooga FEK kasutamine.

Käesolevas artiklis analüüsitakse FEK omamürade peamisi põhjusi ning esitatakse aparatuur ja metoodika omamürade uurimiseks.

Uuritud FEK parameetritest esitatakse omamürade energeetiline ekvivalent, omamürade sõltuvus pingest ja müraspektrid. Need parameetrid mõõdeti ühekanalilise stsintillatsioonloendajaga (joon. 1), mis koosnes Soti firma «Nuclear Enterprises» standardsetest blokkidest. 
Müranivoo määrati integraalse diskriminaatoriga, loenduskiirusel $10 \mathrm{imp} / \mathrm{min}$. Müranivoo energeetiline ekvivalent väljendati $\mathrm{NaJ}(\mathrm{Tl})$ skaalas. Joonisel 2 esitatakse möningate FEK omamürade sõltuvus pingest, joonisel 3 - FEK müraspektrid.

Tabelis esitatakse uuritud FEK iseloomustavate andmete kõrval omamürade energeetilise ekvivalendi minimaalsed väärtused ja nendele vastavad pinged.

\section{A. LIIVA, E. ILVES, J.-M. PUNNING}

\section{DAS ENERGETISCHE ÄQUIVALENT DES RAUSCHPEGELS EINIGER SEKUNDARELEKTRONEN-VERVIELFACHER}

Beim Registrieren weicher $\beta$-Strahlung ist es erforderlich, den Rauschpegel der Messanordnung möglichst klein zu halten. Da die Empfindlichkeit des Szintillationszählers hauptsächlich vom Rauschen des Sekundärelektronen-Vervielfachers (SEV) abhängt, is ${ }^{\prime}$ man bestrebt, besonders beim Registrieren energiearmer $\beta$-Strahlung, rauscharme SEV zu verwenden. Hierbei ist zu erwähnen, dass die technischen Date.. einzelner SEV, sogar innerhalb einer und derselben Herstellungsserie, erheblich schwanken. Daher werden in den Laboratorien diejenigen SEV aus der Produktionsserie ausgewählt (in einigen Fällen tun es die Herstellerfirmen, z. B. EMI), die für Szintillationsmessungen besonders geeignet sind.

In vorliegender Arbeit wird die im Institut für Zoologie und Botanik der Akademie der Wissenschaften der Estnischen SSR eingeführte Methodik beschrieben, die es ermöglicht, aus den vorhandenen SEV verhältnismässig schnell die besten Exemplare auszuwählen.

Es wurden folgende Parameter der SEV untersucht:

1) encrgetisches Äquivalent des Rauschpegels;

2) Abhängigkeit des Rauschpegels von der Spannung am SEV-Teil;

3) Rauschspektren.

Alle Messungen wurden mit einem Einkanal-Szintillationszähler durchgeführt (Abb. 1), welcher aus einem elektronenstabilisierten Hochspannungsgerät (Typ NE 5302), einem Vorverstärker (Typ NE 5202 A), einem Hauptverstärker (Typ NE 5202) und einem Amplituden-Analysator (Typ NE 5102) zusammengestellt wurde. Stabilisierung der Netzspannung erfolgte mittels eines elektronischen Stabilisators ST-2000.

Der Wert des Rauschpegels wurde für jedes SEV-Exemplar, bei der Diskriminatorspannung $10 \mathrm{~V}$, mittels des Hauptverstärkers auf $10 \mathrm{Imp}$. pro Minute eingestellt und das energetische Äquivalent des Rauschpegels in der Skala NaJ (Tl) ausgedrückt (spektrometrischer Kristall, mit den Abmessungen $30 \times 10 \mathrm{~mm}$ ). Zur Energiemessung wurde das Maximum der Fotolinie des $\mathrm{Eu}^{155}(\mathrm{E}=87 \mathrm{KeV})$ verwendet.

In Abb. 2 sind für einige SEV die Werte des Rauschpegels in Abhängigkeit von der Spannung am SEV-Teil angegeben.

Abb. 3 zeigt die Rauschspektren einiger SEV.

In der Tabelle sind neben den Daten der untersuchten SEV die minimalen Werte des Rauschpegels und die ihnen entsprechenden Spannungen angegeben. 